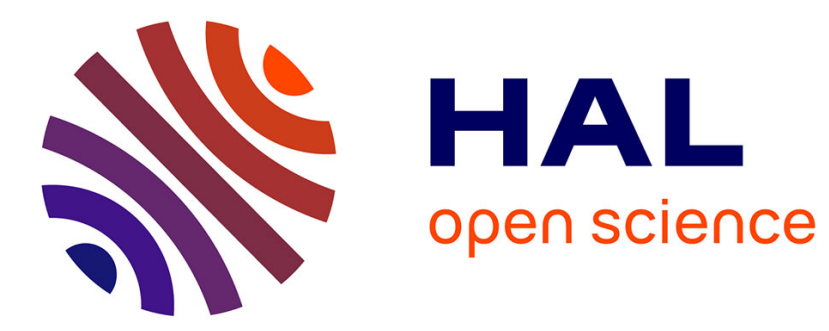

\title{
Controllability Analysis of Quantum Systems Immersed within an Engineered Environment
}

Andreea Grigoriu, Herschel Rabitz, Gabriel Turinici

\section{To cite this version:}

Andreea Grigoriu, Herschel Rabitz, Gabriel Turinici. Controllability Analysis of Quantum Systems Immersed within an Engineered Environment. Journal of Mathematical Chemistry, 2013, 51 (6), pp.1548-1560. 10.1007/s10910-013-0163-6 . hal-00696546v3

\section{HAL Id: hal-00696546 \\ https://hal.science/hal-00696546v3}

Submitted on 18 Nov 2012

HAL is a multi-disciplinary open access archive for the deposit and dissemination of scientific research documents, whether they are published or not. The documents may come from teaching and research institutions in France or abroad, or from public or private research centers.
L'archive ouverte pluridisciplinaire HAL, est destinée au dépôt et à la diffusion de documents scientifiques de niveau recherche, publiés ou non, émanant des établissements d'enseignement et de recherche français ou étrangers, des laboratoires publics ou privés. 


\title{
CONTROLLABILITY ANALYSIS OF QUANTUM SYSTEMS IMMERSED WITHIN AN ENGINEERED ENVIRONMENT*
}

\author{
A. GRIGORIU ${ }^{\dagger}$, H. RABITZ ${ }^{\ddagger}$, AND G. TURINICI $^{\S}$
}

\begin{abstract}
The problem of controllability of quantum systems interacting with an engineered environment, whose dynamics are described by a non-Markowian master equation is addressed. The manipulations of the dynamics is realized with both a laser field and a tailored non-equilibrium, and generally time-dependent, state of the surrounding environment. Lie algebra theory is used to characterize the structures of the reachable state sets and to prove controllability. The theoretical results are supported by examples.
\end{abstract}

Key words. quantum control; controllability of non-Markovian equations; Lie group controllability; controllability in a non-compact Lie group

AMS subject classifications. 81Q93, 93B05

1. Introduction. Since the first successful laboratory experiments obtained in the 1990s [5, 15], the control of quantum systems using laser fields has been subject to significant developments ( $[5,9,15,17,24]$ etc.). An important part of the associated theoretical work has been devoted to the investigation of closed quantum systems with unitary dynamics. However, realistic physical situations include circumstances where the quantum system is not isolated, but interacting with an environment (e.g., a molecule in a solvent). For these type of systems, also called open quantum systems, one of the main characteristics is that the dynamics is non-unitary. Generic open systems may be difficult to control because the environment can contain irreversible dynamics that fights against control mechanisms. We consider in this work a particular situation when the environment can be engineered i.e. its characteristics chosen at will. The resulting circumstance is not a general open quantum system but a large closed system containing a small part is of interest to the experimenter. The mathematical formulation will be identical to that used to describe an open system except that the environment is considered controllable.

For weak system-environment coupling the dynamics of the system is described by a Markovian equation involving the reduced density matrix $\rho$

$$
\frac{d}{d t} \rho(t)=\mathcal{L} \rho(t)
$$

with the generator $\mathcal{L}$ in Lindblad form $($ see $[8,12,13])$ :

$$
\mathcal{L} \rho=-i[H, \rho]+\sum_{i} \gamma_{i}\left(L_{i} \rho L_{i}^{*}-\frac{1}{2} L_{i}^{*} L_{i} \rho-\frac{1}{2} \rho L_{i} L_{i}^{*}\right),
$$

where the first term represents the unitary part of the system dynamics, with $H$ the Hamiltonian of the system. The second term describes the dissipative dynamics, also referred as the dissipator, dependent upon the Lindblad operators $L_{i}$. The time independent coefficients $\gamma_{i}$ represent coupling to the environment, functioning as the relaxation rates for different decay modes of the open system. In the following for any $X$, we denote by $X^{*}$ its adjoint.

For cases of strong system-environment coupling, structured and finite reservoirs, and/or low temperatures one often encounters non-Markovian processes, implying that the dynamics is governed by significant memory effects. A systematic approach to non-Markovian dynamics is provided

\footnotetext{
*This work was supported by "Agence Nationale de la Recherche" (ANR), Projet Blanc EMAQS number ANR2011-BS01-017-01, US-Department of Energy and Army Research Office.

${ }^{\dagger}$ Laboratoire Jacques-Louis Lions, Université Paris-Diderot. 175, rue du Chevaleret, 75013 PARIS, FRANCE(grigoriu@ljll.univ-paris-diderot.fr).

${ }^{\ddagger}$ Frick Laboratory, Department of Chemistry, Princeton, NJ 08544, USA (hrabitz@princeton.edu).

$\S$ CEREMADE, Université Paris Dauphine, Pl. du Mal de Lattre de Tassigny, 75016 PARIS, FRANCE(gabriel.turinici@dauphine.fr).
} 
By projection operator techniques $[14,18,26]$. These techniques can lead to integro-differential equations for the reduced density matrix, through application of the Nakajima-Zwanzig projection operator technique. If a time-convolution free projection operator technique $[4,6,23]$ is applied, one can deduce an approximate or even exact non-Markovian, first-order differential equation for the open system reduced density matrix $\rho$ :

$$
\frac{d}{d t} \rho(t)=\mathcal{K}(t) \rho(t)
$$

The non-Markovian character of this equation is reflected by the fact that its generator $\mathcal{K}$ depends explicitly on time and does not have the Lindblad form above.

If we require that the density matrix be Hermitian and its trace constant, the most general form of the generator $\mathcal{K}(t)$ is given by $[6,13]$ :

$$
\mathcal{K}(t) \rho=-i[H(t), \rho]+\sum_{i} \gamma_{i}(t)\left(A_{i}(t) \rho A_{i}^{*}(t)-\frac{1}{2} A_{i}(t)^{*} A_{i}(t) \rho-\frac{1}{2} \rho A_{i} A_{i}^{*}\right)
$$

where the Hamiltonian $H(t)$, the operators $A_{i}(t)$ and the relaxation rates $\gamma_{i}(t)$ depend on time.

If the relaxation rates $\gamma_{i}(t)$ are positive functions, the generator $\mathcal{K}(t)$ is in Lindblad form for each fixed $t \geqslant 0$. Such processes may be called time-dependent Markovian although the corresponding dynamical map do not lead to a quantum dynamical semigroup [7]. However if the relaxation rates become negative in certain time intervals (see examples of negative rates $\gamma_{i}$ in $[6,7])$, the master equation in no longer in Lindblad form and the generator is not completely positive. Therefore non-Markovian character is also related to the emergence of negative rates.

Given the diversity of models that describe the quantum system-environment interaction, the problem manipulating them becomes difficult to address, especially since environment is usually composed of a large number of degrees of freedom which are difficult to control. Despite that, research on control of such systems is strongly motivated by a large number of applications including quantum computing [10], laser cooling, quantum reservoir engineering, management of decoherence, chemical reactions and energy transfer in molecules [22].

Optimal control by a tailored non-equilibrium, and generally time-dependent, state of the surrounding environment has been addressed in the literature (see [20] for more details). The master equation for the system interacting with an environment, characterized by its distribution function $n_{\mathrm{k}}(t)$ is given by

$$
\frac{d}{d t} \rho(t)=-i[H, \rho(t)]+\sum_{\omega} \gamma_{\omega}\left(n_{\mathrm{k}}(t)\right)\left(A_{\omega} \rho A_{\omega}^{*}-\frac{1}{2} A_{\omega}^{*} A_{\omega} \rho-\frac{1}{2} \rho A_{\omega}^{*} A_{\omega}\right)
$$

where the coefficients $\gamma_{\omega}(t) \geqslant 0$ determine the transitions rates between energy levels with transition frequencies $\omega$.

Control by lasers fields normally affects the system through Hamiltonian evolution and transforms pure states into pure states, while control by the environment (i.e., control by $\gamma(t)$ ) affects a system through dissipative dynamics and can be used to steer the system from a pure or a mixed state into mixed and in some cases pure states (a familiar example is the cooling of a thermalized quantum system, which requires coupling to a reservoir). This type of incoherent control by the environment may be combined with coherent fields to allow for simultaneous control through both the Hamiltonian and dissipative parts of the system dynamics.

The equation for a system that simultaneously interacts with an electromagnetic field $\epsilon(t)$ and an environment described by a function $\gamma(t) \geqslant 0$ generally has the form [20]:

$$
\frac{d}{d t} \rho(t)=-i\left[H_{0}+H_{e f f}+\epsilon(t) H_{1}, \rho(t)\right]+\sum_{i} \gamma_{i}(t)\left(A_{i} \rho A_{i}^{*}-\frac{1}{2} A_{i}^{*} A_{i} \rho-\frac{1}{2} \rho A_{i}^{*} A_{i}\right)
$$


where $H_{0}$ is the Hamiltonian describing the coherent part of the dynamics, $H_{\text {eff }}$ is the effective Hamiltonian describing the unitary part of the system-environment interaction and $H_{1}$ is the dipole moment operator describing the interaction between the system and the field $\epsilon(t)$.

Although the notion of simultaneous control by both a laser and by the environment has been mentioned in the literature [20], a theoretical analysis of the controllability has not been investigated yet. The goal of this paper is to propose such an analysis in a generic situation. A fundamental question involving the system above is whether for any pair of states $\rho_{i}$ and $\rho_{f}$, both positive semidefinite, Hermitian, $\operatorname{tr}\left(\rho_{i}\right)=\operatorname{tr}\left(\rho_{f}\right)>0$, a control $(\epsilon(t), \gamma(t))$ exists such that $\rho(0)=\rho_{i}$ and $\rho(T)=\rho_{f}$ for some $T>0$. If the answer is positive the system is called controllable. Since $\gamma(t)$ may also take negative values, the present work addresses the controllability analysis for situations when both $\epsilon(t)$ and $\gamma(t)$ are arbitrary real numbers. Under this hypothesis the property of complete positivity of the density matrix is lost; moreover with dynamics being non-Markovian. Note that if physical considerations require $\gamma(t)$ to have further specific properties the results of the analysis will not apply, but a practical proposal is to impose the constraints in the numerical simulations by penalization, leading to an optimal control framework.

Thus we suppose that the system follows a first-order differential equation (1.6) for the reduced density matrix $\rho$. The generator for the above equation is time dependent and has the same form as $\mathcal{K}(t)$, previously defined by equation (1.4). Our main goal is to analyze the controllability (as defined above) of the equation (1.6).

Another motivation for this analysis is given by systems that are not controllable in the isolated setting $(\gamma(t)=0)$; one direction on addressing controllability can rely on the use of specially tailored environments, characterized by $\gamma(t)$, applied through the dissipative part of the dynamics. It should be noted, however, that the assumption $\gamma(t) \in \mathbb{R}$ does not imply that the propagation of an arbitrary positive matrix at time $t$ with the help of the master equation (1.6) necessarily leads to a positive matrix for future times. It is important to mention that although we want to introduce results as close as possible to physical circumstances, the complexity of the theoretical problems addressed in the framework of controllability of quantum systems interacting with an environment is a complex topic for which we have a partial analysis.

The balance of the paper is as follows: in Section 2 we introduce the general framework, the main notation together with a short review of Lie algebra controllability results previously introduced in the literature. In Section 3 we characterize the reachable sets and we prove two controllability results. The last section is dedicated to numerical computations.

2. The problem. We consider a quantum system interacting with an environment. The system is manipulated by both a laser field of intensity $\epsilon(t)$ and a tailored non-equilibrium, and generally time-dependent rate, $\gamma(t)$ depending on the state of the surrounding environment; the dynamics is described by equation (1.6). We suppose that the quantum dynamics takes place in a finite dimensional space (e.g., either intrinsically so or because a suitable large basis set approximation has been chosen). Thus, $\rho(t)$ is a $N \times N$ complex matrix for some integer $N>0$, $H_{0}, H_{\text {eff }}$ and $H_{1}$ are real $N$-dimensional symmetric matrices (thus Hermitian), $A_{i}$ is an arbitrary $N$-dimensional matrix and $\epsilon, \gamma$ are real time dependent functions.

In the following, we study the controllability (see the definition above) of the $N$-dimensional equation (1.6). Without loss of generality we consider throughout the paper only the circumstance when all $\gamma_{i}$ are null except $\gamma_{1}$. This assumption is not restrictive since the same general results can be obtained for the case when more then one $\gamma$ is non-null. Since the effective Hamiltonian $H_{e f f}$ is assumed to commute with $H_{0}$ we can redefine $H_{0}$ to be $H_{0}+H_{\text {eff }}$ and omit $H_{\text {eff }}$ in the sequel. Therefore, under the above hypothesis equation (1.6) becomes:

$$
\frac{d}{d t} \rho=-i\left[H_{0}, \rho\right]-i \epsilon(t)\left[H_{1}, \rho\right]+\gamma(t)\left(A \rho A^{*}-\frac{1}{2} \rho A^{*} A-\frac{1}{2} A^{*} A \rho\right) .
$$

In the following, for simplicity, we use the notation $\mathcal{K}$ for the generator of the equation (2.1)

$$
\mathcal{K}(t) \rho=-i\left[H_{0}, \rho\right]-i \epsilon(t)\left[H_{1}, \rho\right]+\gamma(t)\left(A \rho A^{*}-\frac{1}{2} \rho A^{*} A-\frac{1}{2} A^{*} A \rho\right) .
$$


Aspects on controllability of open quantum systems have been addressed in the literature mostly for systems (2.1) with $\gamma$ positive and constant. The problem of kinematic state controllability (KSC) of open quantum systems whose dynamics are represented by Kraus maps has been considered in [25]. The behavior of a Markovian master equation for a $N$-level quantum mechanics system driven by a coherent control field $\epsilon(t)$ is analyzed $[2,3]$. It is shown that the system can be accessible, but neither small-time controllable nor controllable in finite time.

A deterministic two stage method for engineering arbitrary pure and mixed states for quantum systems subject to the Markovian evolution is proposed in [19]. In the first stage, the system evolves under the action of a suitable optimal incoherent control. In the second stage, the system evolves under the action of a suitable coherent laser control. But in that work the focus in on the control of the dissipative regime i.e. the control by the coherent part (the laser of intensity $\epsilon(t)$ is already supposed complete), while here we on the contrary want to use $\gamma(t)$ to bring supplementary control in addition to that of $\epsilon(t)$.

In this paper we use the Lie algebraic approach (cf. $[1,11,21])$ to analyze the controllability of quantum systems those dynamics is described by equation (2.1). An advantage of this technique is that it also provides an explicit controllability criteria, which can be verified using numerical computations.

As will be seen in the sequel, controllability analysis of systems defined by (2.1) must deal with the loss of compactness that arises from the non-Hermitian nature of the generator $\mathcal{K}$ defined by (2.2). Another problem is that we do not know a priori the structure of the reachable states, identifying the Lie group remains a task to be investigated.

2.1. Background on controllability on Lie groups. Let us consider a connected but not necessarily compact Lie group $G$ with Lie algebra $L(G)$ and control system

$$
\frac{d X}{d t}(t)=\mathbb{X}_{0}(X(t))+\sum_{i=1}^{m} u_{i}(t) \mathbb{X}_{i}(X(t))
$$

where $\mathbb{X}_{0}$ and $\mathbb{X}_{i}$ are right-invariant vector fields on $G$. If necessary we will denote this solution $X(t ; u ; Y)$ to indicate its dependence on time, controls and initial state $Y$. Consider the set of all reachable states from $Y$ at time $t$ :

$$
\mathcal{R}^{t}(Y)=\{X(t ; u ; Y) \mid X(t ; u ; Y) \text { solution of }(2.3), X(0 ; u ; Y)=Y\} .
$$

It follows to see that

$$
\mathcal{R}^{t}(Y)=\mathcal{R}^{t}(e) Y
$$

where we denote by $e$ the identity of the Lie group $G$; thus, describing the set $\mathcal{R}^{t}(e) Y$ allows for completely describing all the other reachable sets. When the final time is not specified, we will use

$$
\mathcal{R}(Y)=\cup_{t \geqslant 0} \mathcal{R}^{t}(Y)
$$

We take the admissible controls $u_{i}(t)$ to be the set of all locally bounded and measurable functions.

Consider $\mathbb{L}$ to be the Lie algebra generated by $X_{0}, X_{1}, \ldots, X_{m}$ and $\mathbb{S}$ its corresponding Lie group (Lie subgroup of $G$ ). We do not assume that $\mathbb{S}$ is compact.

The results proved below build on the following reformulation of a result in [16][Thm 6.6] (to which we refer for further details):

THEOREM 2.1 (Jurdjevic and Sussmann 1972). If there exists a constant control $u=\left(u_{1}, \ldots, u_{m}\right)$ and a sequence of positive numbers $\left\{t_{n}\right\}$ with $t_{n} \geqslant \delta>0$, for some $\delta$, with the property that $\lim _{n \rightarrow \infty} X\left(t_{n}, u, e\right)$ exists and belongs to $\overline{\mathbb{S}}$ (the closure is relative to $\left.\mathbb{S}\right)$ then $\mathcal{R}(e)=\mathbb{S}$. 
3. Controllability. We define the operators $\mathcal{H}_{0}, \mathcal{H}_{1}$ and $\mathcal{T}$ as follows:

$$
\begin{aligned}
\mathcal{H}_{0} & : \rho \longrightarrow-i\left[H_{0}, \rho\right] \\
\mathcal{H}_{1} & : \rho \longrightarrow-i\left[H_{1}, \rho\right] \\
\mathcal{T} & : \rho \longrightarrow A \rho A^{*}-\frac{1}{2} \rho A^{*} A-\frac{1}{2} A^{*} A \rho,
\end{aligned}
$$

and rewrite equation (2.1) as:

$$
\frac{d}{d t} \rho=\mathcal{H}_{0} \rho+\epsilon(t) \mathcal{H}_{1} \rho+\gamma(t) \mathcal{T} \rho
$$

We introduce the sets of matrices:

$$
\Gamma_{N}=\left\{Z \in \mathbb{C}^{N \times N} \mid Z=Z^{*}\right\}, \Gamma_{N}^{0}=\left\{Z \in \mathbb{C}^{N \times N} \mid Z=Z^{*}, \operatorname{tr}(Z)=0\right\},
$$

and recall that $\Gamma_{N}\left(\right.$ respectively $\left.\Gamma_{N}^{0}\right)$ has dimension $N^{2}$ (respectively $\left.N^{2}-1\right)$ when seen as a vector space over $\mathbb{R}$.

Note that, denoting by $a d_{x}$ the map $y \mapsto a d_{x}(y)=[x, y]$ (the adjoint representation), we also have

$$
\mathcal{H}_{0}=a d_{-i H_{0}}, \mathcal{H}_{1}=a d_{-i H_{1}} .
$$

Also, when $A=A^{*}$ then $\mathcal{T}=-\frac{1}{2} a d_{A} \circ a d_{A}$. A simple computation indicates that for any $Z \in$ $\Gamma_{N}: \mathcal{H}_{0}(Z), \mathcal{H}_{1}(Z), \mathcal{T}(Z) \in \Gamma_{N}$. Moreover all three operators are linear. Thus $\mathcal{H}_{0}, \mathcal{H}_{1}, \mathcal{T} \in$ $\operatorname{Lin}\left(\Gamma_{N}, \Gamma_{N}\right)$, the space of linear operators from $\Gamma_{N}$ to $\Gamma_{N}$ (this also results from Eqn (3.4)). Also note that for any matrix $Z$ :

$$
\begin{aligned}
\operatorname{tr}\left(\mathcal{H}_{0} Z\right) & =\operatorname{tr}\left(-i\left[H_{0}, Z\right]\right)=0 \\
\operatorname{tr}\left(\mathcal{H}_{1} Z\right) & =\operatorname{tr}\left(-i\left[H_{1}, Z\right]\right)=0 \\
\operatorname{tr}(\mathcal{T} Z) & =\operatorname{tr}\left(A Z A^{*}-\frac{1}{2} Z A^{*} A-\frac{1}{2} A^{*} A Z\right)=0 .
\end{aligned}
$$

Thus we also have $\mathcal{H}_{0}, \mathcal{H}_{1}, \mathcal{T} \in \operatorname{Lin}\left(\Gamma_{N}^{0}, \Gamma_{N}^{0}\right)$. In particular the trace of $\rho$ will not change during the evolution. Moreover as we will see below, when $A$ is Hermitian another conservation law exists: if $\rho(0)=\lambda I$ for some $\lambda \in \mathbb{R}$ then $\rho(t)=\lambda I$ for all $t \geq 0$. This motivates the following definition:

DeFINITION 3.1. The evolution (3.2) is density matrix controllable if for any positive semidefinite Hermitian matrices $\rho_{i}$ and $\rho_{f}$ with $\operatorname{tr}\left(\rho_{i}\right)=\operatorname{tr}\left(\rho_{f}\right)>0$ there exists a time $t \geq 0$ and locally bounded measurable controls $\epsilon(\cdot), \gamma(\cdot)$ such that the solution of the evolution equation (2.1) starting at 0 from $\rho_{i}$ reaches $\rho_{f}$ at time $t$. When $A=A^{*}$ we suppose $\rho_{i} \neq \frac{\operatorname{tr}\left(\rho_{i}\right)}{N} I d, \rho_{f} \neq \frac{\operatorname{tr}\left(\rho_{f}\right)}{N} I d$.

The standard Lie group setting for investigating the control of an isolated quantum system works with the Lie group of unitary (or special unitary) matrices $U(N)$ (or $S U(N)$ ). Here, on the contrary, we work on the group of one-to-one transformations of $\Gamma_{N}$ to $\Gamma_{N}$ (or the same for $\Gamma_{N}^{0}$ ). As a numerical illustration of the difference, the dimension of $U(N)$ as a real Lie group is $N^{2}$ while the dimension of the one-to-one transformations from $\Gamma_{N}$ to itself is $N^{4}$.

We investigate controllability results in two distinct situations: when the matrix $A$ is Hermitian or not.

REMARK 3.1. The particular case of A being Hermitian corresponds to unital operator $\mathcal{T}$, i.e. $\mathcal{T} I=0$, which is a situation frequently addressed in quantum information processing.

3.1. Situation I: Hermitian operator $A$. Let us now consider the connected Lie group $G_{1}$ of one-to-one linear transformations of $\Gamma_{N}^{0}$ that contains the identity operator. The canonical notation of this group is $G L^{+}\left(\Gamma_{N}^{0}\right)$. As we do not impose any particular structure on $\Gamma_{N}^{0}$ except that of vector space, $\Gamma_{N}^{0}$ is isomorphic to $\mathbb{R}^{N^{2}-1}$; thus the Lie group $G L^{+}\left(\Gamma_{N}^{0}\right)$ is isomorphic to $G L^{+}\left(N^{2}-1\right)$, the Lie group of invertible matrices of dimension $\left(N^{2}-1\right) \times\left(N^{2}-1\right)$ with positive 
Geterminant. In particular its dimension is $\left(N^{2}-1\right)^{2}$. We know that $G_{1}$ is connected but not compact. We will denote by $\operatorname{Lie}\left(G_{1}\right)$ the Lie algebra of $G_{1}$ which is therefore $\operatorname{Lin}\left(\Gamma_{N}^{0}, \Gamma_{N}^{0}\right)$.

Denote by $\mathcal{H}_{0}^{G_{1}}$ the element of $\operatorname{Lie}\left(G_{1}\right)$ that is constructed from $\mathcal{H}_{0}$ (and the same for $\mathcal{H}_{1}$ and $\mathcal{T}$ ). We associate to the evolution equation (3.2) the following evolution equation on the group $G_{1}$ :

$$
\begin{aligned}
\frac{d}{d t} X(t) & =\left(\mathcal{H}_{0}^{G_{1}}+\epsilon(t) \mathcal{H}_{1}^{G_{1}}+\gamma(t) \mathcal{T}^{G_{1}}\right) X(t), \\
X(t=0) & =X_{0} .
\end{aligned}
$$

We will also write $X\left(t ; \epsilon, \gamma ; X_{0}\right)$ when we will need to make explicit the dependence on the parameters; of course $X\left(t ; \epsilon, \gamma ; X_{0}\right)$ belongs to the Lie group $G_{1}$. Then by definition $\bar{\rho}(t)=X(t ; \epsilon, \gamma ; e) \bar{\rho}(0)$ (here $e$ is the identity of the Lie group $G_{1}$ ).

THEOREM 3.2. If the Lie algebra Lie $\left\{\mathcal{H}_{0}^{G_{1}}, \mathcal{H}_{1}^{G_{1}}, \mathcal{T}^{G_{1}}\right\} \subset \operatorname{Lie}\left(G_{1}\right)$ generated by $\left\{\mathcal{H}_{0}^{G_{1}}, \mathcal{H}_{1}^{G_{1}}, \mathcal{T}^{G_{1}}\right\}$ has dimension $\left(N^{2}-1\right)^{2}$ (as a vector space over the real numbers), then the system (3.2) is density matrix controllable.

Proof. Without loss of generality we can suppose that $\operatorname{tr}\left(H_{0}\right)=0$ and $\operatorname{tr}\left(H_{1}\right)=0$.

As a side remark, note that since $A$ is Hermitian we can show by computation that $\mathcal{T}$ is a Hermitian operator from $\Gamma_{N}^{0}$ to itself i.e., $\mathcal{T}^{*}=\mathcal{T}$. However $\mathcal{H}_{0}$ and $\mathcal{H}_{1}$ are skew-Hermitian as operators from $\Gamma_{N}^{0}$ to itself.

We consider the following change of variables:

$$
\bar{\rho}(t)=\rho(t)-\frac{\operatorname{tr}(\rho(0))}{N} I,
$$

which implies that $\bar{\rho}(0) \in \Gamma_{N}^{0}$. However this does not imply directly that $\bar{\rho}(t) \in \Gamma_{N}^{0}$ for all $t>0$. To prove this one recalls that $A$ is Hermitian and $\mathcal{H}_{0}, \mathcal{H}_{1}, \mathcal{T} \in \operatorname{Lin}\left(\Gamma_{N}^{0}, \Gamma_{N}^{0}\right)$; after invoking the Frobenius theorem for the equation:

$$
\dot{\bar{\rho}}=-i[H, \bar{\rho}]+\mathcal{T}(\bar{\rho})
$$

one obtains that $\bar{\rho}(t) \in \Gamma_{N}^{0}$ for all $t \geq 0$.

From the above relations we conclude that $\mathcal{H}_{0}(\rho), \mathcal{H}_{1}(\rho)$ and $\mathcal{T}(\rho)$ belong to $\operatorname{Lin}\left(\Gamma_{N}^{0}, \Gamma_{N}^{0}\right)$.

The central question is to characterize $\mathcal{R}(e)$ for the system (3.5). We will use Theorem 2.1 with $G=G_{1}$. Then $\mathbb{L}=\operatorname{Lie}\left\{i \mathcal{H}_{0}^{G_{1}}, i \mathcal{H}_{1}^{G_{1}}, \mathcal{T}^{G_{1}}\right\}$ is the Lie algebra generated by $\mathcal{H}_{0}^{G_{1}}, \mathcal{H}_{1}^{G_{1}}$ and $\mathcal{T}^{G_{1}}$.

Recall that $\mathbb{L} \subset \operatorname{Lie}\left(G_{1}\right)$ and $\operatorname{dim}_{\mathbb{R}}\left(\operatorname{Lie}\left(G_{1}\right)\right)=\operatorname{dim}_{\mathbb{R}}\left(\operatorname{Lin}\left(\Gamma_{N}^{0}, \Gamma_{N}^{0}\right)\right)=\left(N^{2}-1\right)^{2}$ (dimension as vector space over $\mathbb{R})$. By hypothesis $\operatorname{dim}_{\mathbb{R}}(\mathbb{L})=\left(N^{2}-1\right)^{2}$ thus $\mathbb{L}=\operatorname{Lie}\left(G_{1}\right)$ and $\mathbb{S}=G_{1}$. Take now $\epsilon=0, \gamma=0$, then equation (3.5) becomes:

$$
\begin{aligned}
\frac{d}{d t} X(t) & =\mathcal{H}_{0}^{G_{1}} X(t), \\
X(t=0) & =e,
\end{aligned}
$$

with solution $X(t)=e^{t \mathcal{H}_{0}^{G_{1}}}$; take a sequence of positive numbers $\left\{t_{n}\right\}$ with $t_{n} \geqslant \delta>0$ for some $\delta$ (strictly positive, arbitrary but fixed); we need to prove that

$$
\lim _{n \rightarrow \infty} e^{t_{n} \mathcal{H}_{0}^{G_{1}}} \in \overline{\mathbb{S}}
$$

But, $\mathcal{H}_{0}^{G_{1}}$ is a skew-Hermitian map, which means that $e^{t_{n} \mathcal{H}_{0}^{G_{1}}}$ belongs to the group $S O\left(\Gamma_{N}^{0}\right)$ of the special orthogonal transformations of $\Gamma_{N}^{0}$; it is known that $S O\left(\Gamma_{N}^{0}\right)$ is the maximal compact subgroup of $G_{1}=G L^{+}\left(\Gamma_{N}^{0}\right)$.

By compactness we obtain that up to extracting a subsequence $\lim _{n \rightarrow \infty} e^{t_{n} \mathcal{H}_{0}^{G_{1}}}$ exists and belongs to $\overline{\mathbb{S}}$. Therefore $\mathcal{R}(e)=\mathbb{S}$. In particular $X$ can reach any one-to-one transformation from 
$\Gamma_{N}^{0}$ to itself. For any $\rho_{i}$ and $\rho_{f}$ with $\operatorname{tr}\left(\rho_{i}\right)=\operatorname{tr}\left(\rho_{i}\right), \rho_{i}-\frac{\operatorname{tr}\left(\rho_{i}\right)}{N} I \neq 0$ and $\rho_{f}-\frac{\operatorname{tr}\left(\rho_{f}\right)}{N} I \neq 0$ we can find a transformation to map the non-null vector $\rho_{i}-\frac{\operatorname{tr}\left(\rho_{i}\right)}{N} I \in \Gamma_{N}^{0}$ to the non-null vector $\rho_{f}-\frac{\operatorname{tr}\left(\rho_{f}\right)}{N} I=\rho_{f}-\frac{\operatorname{tr}\left(\rho_{i}\right)}{N} I \in \Gamma_{N}^{0}$ i.e., we have controllability for $\bar{\rho}$ thus for $\rho$.

REMARK 3.2. Following the proof of Theorem 3.2 we can also conclude that the set of all density matrices reachable from $\rho_{i}\left(\right.$ with $\left.\rho_{i} \neq \frac{\operatorname{tr}\left(\rho_{i}\right)}{N} I\right)$ with dynamics described by equation (3.2), for $A$ being a Hermitian operator, is

$$
\left(\rho_{i}+\Gamma_{N}^{0}\right) \backslash\left\{\frac{\operatorname{tr}\left(\rho_{i}\right)}{N} I\right\}=\left\{\rho_{i}+Z \mid Z \in \Gamma_{N}^{0}, Z \neq \frac{\operatorname{tr}\left(\rho_{i}\right)}{N} I-\rho_{i}\right\} .
$$

3.2. Situation II: arbitrary operator $A$. Let us now consider the connected Lie group $G_{2}$ of one-to-one linear transformations of $\Gamma_{N}$ that contains the identity operator and preserves the trace:

$$
G_{2}:=\left\{X \in G L^{+}\left(\Gamma_{N}\right) \mid \operatorname{tr}(X(Z))=\operatorname{tr}(Z), \forall Z \in \Gamma_{N}\right\} .
$$

By construction of $\Gamma_{N}$ we only have a canonical linear space structure, thus $\Gamma_{N}$ can be identified with $\mathbb{R}^{N^{2}}$; and thus $G_{2}$ is isomorphic with

$$
\left\{X \in G L\left(N^{2}\right) \mid \operatorname{det}(X) \geq 0, \operatorname{tr}(X(Z))=\operatorname{tr}(Z), \forall Z \in \mathbb{R}^{N^{2}}\right\} .
$$

We know that $G_{2}$ is connected but not compact. We will denote by $\operatorname{Lie}\left(G_{2}\right)$ the Lie algebra of $G_{2}$. Note that if $\frac{d}{d t} \rho(t)=M(\rho(t))$ then $\rho(t)=\exp (M t) \rho(0)$. If $\operatorname{tr}(\rho(t))=$ const it implies $\frac{d}{d t} \operatorname{tr}(\rho(t))=0$. Since $\frac{d}{d t} \operatorname{tr}(\rho(t))=\operatorname{tr}\left(\frac{d}{d t} \rho(t)\right)$ it follows:

$$
\operatorname{tr}(M(\rho(t)))=0, \forall \rho(t) \in \Gamma_{N} .
$$

Since $\operatorname{tr}(\rho)$ is a linear operation and if $\rho$ is viewed as a vector in $\mathbb{R}^{N^{2}}$ then $\operatorname{tr}(\rho)=\langle\alpha, \rho>$ with $\alpha$ a vector with " 1 " on the positions corresponding to $\rho_{i i}, i=1, \ldots, N^{2}$ and zero elsewhere. In this case the Lie algebra is thus isomorphic with the set (endowed with its canonical Lie algebra structure):

$$
\left\{M \in \mathbb{R}^{N^{2} \times N^{2}} \mid M^{T} \alpha \equiv 0_{\mathbb{R}^{N^{2}}} \text { i.e. } \sum_{j=1}^{N^{2}} M_{j i} \alpha_{j}=0, \forall i=1, \ldots, N^{2}\right\}
$$

We have thus $N^{2}$ constraints on $\mathbb{R}^{N^{2} \times N^{2}}$, that means the dimension over $\mathbb{R}$ of the Lie algebra is $\left(N^{2}-1\right) N^{2}$.

Denote by $\mathcal{H}_{0}^{G_{2}}$ the element of $\operatorname{Lie}\left(G_{2}\right)$ that is constructed from $\mathcal{H}_{0}$ (and the same for $\mathcal{H}_{1}$ and $\mathcal{T}$ ). We associate to the evolution equation (3.2) the following evolution equation on the group $G_{2}$ :

$$
\begin{aligned}
\frac{d}{d t} X(t) & =\left(\mathcal{H}_{0}^{G_{2}}+\epsilon(t) \mathcal{H}_{1}^{G_{2}}+\gamma(t) \mathcal{T}^{G_{2}}\right) X(t), \\
X(t=0) & =X_{0} .
\end{aligned}
$$

We will also write $X\left(t ; \epsilon, \gamma ; X_{0}\right)$ when we will need to make explicit the dependence on the parameters; of course $X\left(t ; \epsilon, \gamma ; X_{0}\right)$ belongs to the Lie group $G_{2}$. Then by definition $\bar{\rho}(t)=X(t ; \epsilon, \gamma ; e) \bar{\rho}(0)$.

THEOREM 3.3. If the Lie algebra Lie $\left\{\mathcal{H}_{0}^{G_{2}}, \mathcal{H}_{1}^{G_{2}}, \mathcal{T}^{G_{2}}\right\}$ generated by $\left\{\mathcal{H}_{0}^{G_{2}}, \mathcal{H}_{1}^{G_{2}}, \mathcal{T}^{G_{2}}\right\}$ has dimension $\left(N^{2}-1\right) N^{2}$ (as a vector space over the real numbers) then the system (3.2) is density matrix controllable.

Proof. Without loss of generality we can suppose that $\operatorname{tr}\left(H_{0}\right)=0$ and $\operatorname{tr}\left(H_{1}\right)=0$. We did not yet prove that $G_{2}$ is indeed a Lie group: this results from Cartan's theorem that states that any closed subgroup of a Lie group is a Lie subgroup (we include $G_{2}$ in the group $G L^{+}\left(\Gamma_{N}\right)$ of 
\&ne-to-one transformations on $\Gamma_{N}$ of positive determinant). We will use the same line of proof as in Theorem 3.2 and invoke Theorem 2.1 (this time without any change of variables) for $G=G_{2}$. Then $\mathbb{L}=\operatorname{Lie}\left\{i \mathcal{H}_{0}^{G_{2}}, i \mathcal{H}_{1}^{G_{2}}, \mathcal{T}^{G_{2}}\right\}$ is the Lie algebra generated by $\mathcal{H}_{0}^{G_{2}}, \mathcal{H}_{1}^{G_{2}}$ and $\mathcal{T}^{G_{2}}$.

Recall that $\mathbb{L} \subset \operatorname{Lie}\left(G_{2}\right)$ and $\operatorname{dim}_{\mathbb{R}}\left(\operatorname{Lie}\left(G_{2}\right)\right)=\left(N^{2}-1\right) N^{2}$ (dimension as vector space over $\mathbb{R})$. By hypothesis $\operatorname{dim}_{\mathbb{R}}(\mathbb{L})=\left(N^{2}-1\right) N^{2}$ thus $\mathbb{L}=\operatorname{Lie}\left(G_{2}\right)$ and $\mathbb{S}=G_{2}$. Take now $\epsilon=0, \gamma=0$, then equation (3.15) becomes:

$$
\begin{aligned}
\frac{d}{d t} X(t) & =\mathcal{H}_{0}^{G_{2}} X(t), \\
X(t=0) & =e,
\end{aligned}
$$

with solution $X(t)=e^{t \mathcal{H}_{0}^{G_{2}}}$; take a sequence of positive numbers $\left\{t_{n}\right\}$ with $t_{n} \geqslant \delta>0$ for some $\delta$ (strictly positive, arbitrary but fixed); we need to prove that

$$
\lim _{n \rightarrow \infty} e^{t_{n} \mathcal{H}_{0}^{G_{2}}} \in \overline{\mathbb{S}}
$$

But $\mathcal{H}_{0}^{G_{2}}$ is a skew-Hermitian map which means that $e^{t_{n} \mathcal{H}_{0}^{G_{2}}}$ belongs to the group $S O\left(\Gamma_{N}\right)$ of special orthogonal transformations of $\Gamma_{N}$ which is a subgroup of $G_{2}$. Since $S O\left(\Gamma_{N}\right)$ is compact it implies that up to extracting a subsequence $\lim _{n \rightarrow \infty} e^{t_{n} \mathcal{H}_{0}^{G_{2}}}$ exists and is orthogonal. All orthogonal transformations $e^{t_{n} \mathcal{H}_{0}^{G_{2}}}$ preserve the trace thus $\lim _{n \rightarrow \infty} e^{t_{n} \mathcal{H}_{0}^{G_{2}}}$ exists, is orthogonal and trace preserving and hence an element of $\overline{\mathbb{S}}$. Therefore $\mathcal{R}(e)=\mathbb{S}$. In particular $X$ can reach any trace preserving one-to-one transformation from $\Gamma_{N}$ to itself. For any $\rho_{i}$ and $\rho_{f}$ with $\operatorname{tr}\left(\rho_{i}\right)=\operatorname{tr}\left(\rho_{i}\right)$ we can find a trace preserving one-to-one transformation to map $\rho_{i} \in \Gamma_{N}$ to $\rho_{f} \in \Gamma_{N}$ i.e. we have controllability for $\rho$. $\square$

REMARK 3.3. From the proof of Theorem 3.3 we obtain that the set of all density matrices reachable from $\rho_{i}$ with dynamics described by equation (3.2), for A arbitrary operator, is the set

$$
\rho_{i}+\Gamma_{N}^{0}=\left\{\rho_{i}+Z \mid Z \in \Gamma_{N}^{0}\right\} .
$$

It should be noted that in particular this set contains matrices that are not necessarily positive semidefinite. This means that the control, even between two states that are positive semidefinite may also use intermediary states $\rho(t)$ that are not physical. Should such a circumstance appear in practice, a numerical algorithm that penalizes, e.g. the presence of non-physical states, may be used. The result above indicates that an initial guess that offers perfect control but with a possible non-physical trajectory, can always be subject to such an algorithm.

REMARK 3.4. A straightforward extension is to consider the circumstance when several nonnull controls $\gamma_{2}, \ldots$ are present; the theoretical results can be proved in the same manner.

REMARK 3.5. In this paper two controllability results are proved for $\gamma(t) \in \mathbb{R}$ but not necessarily positive [6]. However physical considerations may impose $\gamma$ to be positive (see [8] for more details). Previous controllability results cannot be used due to the loss of compactness. Therefore the controllability analysis of the system defined by $(2.1)$, for $\gamma(t) \geq 0$, remains for now a conjecture and leads to the question: are Theorems 3.2 and 3.3 true if $\gamma(t)$ takes only positive values?

4. Application. In the following we illustrate the theoretical results introduced in the above section. For this purpose consider two finite-dimensional systems defined by

$$
H_{0}=\left(\begin{array}{ccc}
-4 & 0 & 0 \\
0 & 1 & 0 \\
0 & 0 & 3
\end{array}\right), H_{1}=\left(\begin{array}{ccc}
0 & -2 & 0 \\
-2 & 0 & 0 \\
0 & 0 & 0
\end{array}\right), A=\left(\begin{array}{ccc}
2 & 1 & 3 \\
1 & -1 & 0 \\
3 & 0 & -1
\end{array}\right),
$$

and

$$
H_{0}=\left(\begin{array}{ccc}
-4 & 0 & 0 \\
0 & 1 & 0 \\
0 & 0 & 3
\end{array}\right), H_{1}=\left(\begin{array}{ccc}
0 & -2 & 0 \\
-2 & 0 & 0 \\
0 & 0 & 0
\end{array}\right), A=\left(\begin{array}{ccc}
2 & 1 & 1 \\
1 & -1 & 0 \\
3 & 0 & -1
\end{array}\right)
$$


First note that the system defined by $H_{0}$ and $H_{1}$ alone (i.e., with $A=0$ ) is not controllable as the dimension of the Lie algebra generated by $-i H_{0}$ and $-i H_{1}$ is 4 , short of $3^{2}-1=8$ needed for controllability. Also note that for the system defined by (4.1) $A$ is a symmetric matrix. We want to verify if systems (4.1) and (4.2) are controllable i.e., verify the hypotheses of Theorem 3.2 and Theorem 3.3.

To do so we choose a parameterization such that we can write (3.2) as a linear system

$$
\frac{d}{d t} \tilde{\rho}=-i \tilde{\mathcal{H}}_{0} \tilde{\rho}-i \epsilon(t) \tilde{\mathcal{H}}_{1} \tilde{\rho}+\gamma(t) \tilde{\mathcal{T}} \tilde{\rho}
$$

Numerically $\tilde{\mathcal{H}}_{0}, \tilde{\mathcal{H}}_{1}, \tilde{\mathcal{T}}$ are $N^{2} \times N^{2}$ dimensional matrices and $\tilde{\rho}$ is a $N^{2} \times 1$ vector. For the Hamiltonian part of the dynamics this is known as the Liouville equation in the adjoint representation.

In order to analyze the controllability we need to numerically compute the dimension of the Lie algebra (as subalgebra of $N^{2} \times N^{2}$ matrices) generated by $\left\{i \tilde{\mathcal{H}}_{0}, i \tilde{\mathcal{H}}_{1}, \tilde{\mathcal{T}}\right\}$, which we denote by $\operatorname{Lie}\left\{i \tilde{\mathcal{H}}_{0}, i \tilde{\mathcal{H}}_{1}, \tilde{\mathcal{T}}\right\}$ and verify for $A$ a Hermitian $N$-dimensional matrix if $\operatorname{dim}_{\mathbb{R}}\left(\operatorname{Lie}\left\{i \tilde{\mathcal{H}}_{0}, i \tilde{\mathcal{H}}_{1}, \tilde{\mathcal{T}}\right\}\right)=$ $\left(N^{2}-1\right)^{2}$ or when $A$ is an arbitrary if $\operatorname{dim}_{\mathbb{R}}\left(\operatorname{Lie}\left\{i \tilde{\mathcal{H}}_{0}, i \tilde{\mathcal{H}}_{1}, \tilde{\mathcal{T}}\right\}\right)=\left(N^{2}-1\right) N^{2}$. For the system in (4.1) numerical computations give the result

$$
\operatorname{dim}_{\mathbb{R}}\left(\operatorname{Lie}\left\{i \tilde{\mathcal{H}}_{0}, i \tilde{\mathcal{H}}_{1}, \tilde{\mathcal{T}}\right\}\right)=64
$$

and for the system (4.2)

$$
\operatorname{dim}_{\mathbb{R}}\left(\operatorname{Lie}\left\{i \tilde{\mathcal{H}}_{0}, i \tilde{\mathcal{H}}_{1}, \tilde{\mathcal{T}}\right\}\right)=72 .
$$

We conclude that both systems are controllable.

Acknowledgments. The authors are grateful to Alireza Shabani for useful discussions and comments.

\section{REFERENCES}

[1] C. Altafini, Controllability of quantum mechanical systems by root space decomposition of su(n), J. Math. Phys., 43(5) (2002), pp. 2051-2062.

[2] C. Altafini, Controllability properties for finite dimensional quantum Markovian master equations, J. Math. Phys., 44(6) (2003), p. 2357.

[3] - Coherent control of open quantum dynamical systems, Phys. Rev. A, 70 (2004), p. 062321.

[4] T. Arimitsu and F. Shibata, Expansion formulas in nonequilibrium statistical mechanics, J. Phys. Soc. Jpn., 49 (1980), p. 891.

[5] A. Assion, T. Baumert, M. Bergt, T. Brixner, B. Kiefer, V. Seyfried, M. Strehle, and G. Gerber, Control of chemical reactions by feedback-optimized phase-shaped femtosecond laser pulses, Science, 282 (1998), pp. 919-922.

[6] H.-P. Breuer, Genuine quantum trajectories for non-Markovian processes, Phys. Rev. A, 70 (2004), p. 012106.

[7] H.-P. Breuer, E.-M. Laine, And J. Pillo, Measure for the degree of non-Markovian behavior of quantum processes in open systems, Phys. Rev. Lett., 103 (2009), p. 210401.

[8] H.-P. Breuer and F. Petruccione, The theory of open quantum systems, Clarendon Press, Oxford, 2006.

[9] P. Brumer and M. Shapino, Coherent chemistry: Controlling chemical reactions with lasers., Acc. Chem. Res., 22 (1989), p. 407.

[10] I. L. Chuang, R. Laflamme, P. W. Shor, and W. H. Zurek, Quantum computers, factoring, and decoherence, Science, 270 (1995), pp. 1633-1635.

[11] M. Dahleh, H. Rabitz, V. Ramakrishna, A. Pierce, and M. and Salapaka, Controllability of molecular systems, Phys. Rev. A, 51 (2) (1995), pp. 960-966.

[12] V. Gorini, A. Frigerio, M. Verri, A. Kossakowski, and E. C. G. Sudarshan, Properties of Quantum Markovian Equations, Rep. Math. Phys., 13 (1978), p. 149.

[13] V. Gorini, A. Kossakowski, and E. C. G. Sudarshan, Completely positive dynamical semigroups of N-level systems, J. Math. Phys, 17 (1976), p. 821.

[14] F. HAake, Statistical treatment of open systems, Tracts in Modern Physics, 66 (1973).

[15] R.S. Judson and H. Rabitz, Teaching lasers to control molecules, Phys. Rev. Lett., (1992).

[16] V. Jurdjevic and H. J. Sussmann, Control systems on Lie groups., J. Differ. Equations, 12 (1972), pp. 313329.

[17] R. J. Levis, G.M. MenkiR, And H. Rabitz, Selective bond dissociation and rearrangement with optimally tailored, strong-field laser pulses, Science, 292 (2001), pp. 709-713. 
1.P8] S. Nakajima, On quantum theory of transport phenomena, Prog. Theor. Phys., 20 (1958), pp. 948-959.

[19] A. Pechen, Engineering arbitrary pure and mixed quantum states, Phys. Rev. A, 84 (2011), p. 042106.

[20] A. Pechen and H. Rabitz, Teaching the environment to control quantum systems, Phys. Rev. A, 73 (2006), p. 062102.

[21] H. Rabitz And G. Turinici, Wavefunction controllability in quantum systems, J. Phys.A., 36 (2003), pp. 25652576.

[22] H. Rabitz, G. Turinici, And E. Brown, Control of quantum dynamics: Concepts, procedures and future prospects, in Computational Chemistry, Special Volume (C. Le Bris Editor) of Handbook of Numerical Analysis, vol X, Ph. G. Ciarlet, ed., Elsevier Science B.V., 2003, pp. 833-887.

[23] A. Royer, Combining projection superoperators and cumulant expansions in open quantum dynamics with initial correlations and fluctuating hamiltonians and environments, Phys. Lett. A, 315 (2003), p. 335.

[24] T.C. Weinacht, J. Ahn, And P.H. Bucksbaum, Controlling the shape of a quantum wavefunction, Nature, 397 (1999), pp. 233-235.

[25] R. Wu, A. Pechen, C. BRIF, AND H. Rabitz, Controllability of open quantum systems with Kraus-map dynamics, J. Phys. A, 40 (2007), p. 405681.

[26] R. ZWANZING, Experimental control of the transition from markovian to non-markovian dynamics of open quantum systems, J. Chem. Phys, 33 (1960), p. 1338. 